\title{
Stem Cell Therapy in Anterior Cruciate Ligament (ACL) Injury
}

\author{
Ukhti Jamil Rustiasari \\ Department of Anatomical Pathology \\ Faculty of Medicine \\ Universitas Islam Indonesia \\ Yogyakarta, Indonesia \\ ukhti.rustiasari@uii.ac.id
}

\author{
Muhammad Ikhwan Zein \\ Department of Sport Coaching \\ Faculty of Sports Science \\ Universitas Negeri Yogyakarta \\ Yogyakarta, Indonesia \\ dr_ichwanz@uny.ac.id
}

\begin{abstract}
The incidence of musculoskeletal injuries are the most common injuries that occur in exercise practice. These injuries can involve many different tissues such as muscles, tendons, ligaments, meniscus, bone or cartilage. Anterior cruciate ligament (ACL) tears are the most common athletic injury that occurs in the world, occupies up to $50 \%$ of the incidence of knee injury. Optimal healing process has become a consideration in choosing the most suitable management especially for professional athletes as well as their teams. Many recent studies in the management of musculoskeletal injuries is currently been developed from basic molecular level, experimental studies until clinical application in patients. The application of stem cells therapy becomes a great interest in ACL injury. The ability of stem cells to undergo multilineage differentiation into a variety of cell types is expected to have great potential in the process of repair and regeneration of musculoskeletal injury. The purpose of this review is to summarize the latest developments in the application of stem cell therapy and its safety and effectiveness in ACL injury.
\end{abstract}

Keywords-musculoskeletal injury, anterior cruciate ligament tears, ACL injury, stem cell therapy, safety

\section{INTRODUCTION}

The knee joint is the second location in the body that is most often injured after the ankle. The cruciate ligaments are probably the most famous and one of the most common ligaments that can be damaged. Anterior cranial ligament injury, or ACL injury, is the most common athletic injury that occurs in the world. This ligament is the knee ligaments that break most often in athletes, occupies up to $50 \%$ of the incidence of knee injury [1,2]. The results of a systematic study found that the incidence of ACL in amateur sports groups is generally higher than the incidence of the entire population, but the incidence is lower than that of professional athletes [3].

Ligaments are elastic collagen tissues which generally stabilize and control joint movements. During sports activities, ligaments are the part that gets higher load along with the use of joints and excessive movement [4]. ACL injury can be caused by contact injury, but is more common due to non-contact injury. This condition usually occurs when an athlete in the position of knee hyperextention or suddenly changes his directional line. The circular movements on the knee when the foot rests on the ground or when landing or pushing directly towards the outside of the knee, can cause the rupture of ACL $[1,4]$.
ACL injury may become the end of an athlete's career and may affect his physical activity permanently [2]. This ligament damage can impair the physiological loads and joint movements [4]. Anterior cruciate ligament injury often require surgical repair followed by long-term rehabilitation [2]. Data in the USA estimates that a half of ACL cases require surgical reconstruction [1].

\section{LigAMENT HEALING PROCESS}

Ligament healing is a complicated process which includes the degradation of an old extracellular matrix (ECM) collagen and the formation of a new ECM collagen and its new deposition process. Several studies showed that the ability of intraarticular soft tissue healing is generally lower than in other normal tissue injury. Unlike the medial cruciate ligament (MCL) which has the capacity in healing process and restoring the joint function when a rupture occurs in a short period of time, even in many cases it does not require surgery, injury in the ACL is unable to achieve a satisfactory improvement even with surgery. ACL injuriy often leads to instability of the knee, pain and even develop to other degeneration of joint tissues [5].

The failure of ligament healing process can be affected by a lack of ability in structural protein synthesis which leads to some scar formation or massive degradation of ligamentous when injury occurs. Xie et al showed that in ACL injury, the expression of IL-1 $\beta$ proinflammatory cytokines was higher, leading to higher expression of MMPs and lower LOXs expression than MCL injury. The MMPs enzyme plays a role in ECM ligament degradation, whereas LOXs plays a role in the formation of ECM and its repair process [5].

\section{MANAGEMENT IN ACL INJURY}

Conservative management of ACL injury is performed with rehabilitation which aims to restore knee joint movements and muscle strength. When joint movements and muscle strength have been restored, the activity can be carried out gradually to assess the functional level that has been achieved without causing joint instability. However, in general, conservative rehabilitation alone, provides poor results for patients who want to return to competition. The results of several studies showed that the incidence of instability occured as much as $56-89 \%$ includes pain, swelling and disability. This instability places the meniscus and knee joint cartilage at another risk of injury and only 
about less than $20 \%$ of athletes can return to the competition with this conservative management [6].

At present, the selection of effective therapeutic treatments is very important to achieve a successful cure rate for ACL injuries. Reconstructive surgery in ACL injury is determined individually based on various considerations such as the patient's desire to return to competition at the same level, the age of the patient, the presence of accompanying degenerative changes such as knee osteoarthritis, and the presence of knee joint instability from the examination [6].

In case of ACL injury with a very severe rupture or complete rupture, the choice of surgery is a viable option. At the beginning of the development of surgical techniques, the method of primary reconstruction of ligaments did not provide good clinical results. At present, various reconstruction techniques have been developed to obtain the most effective method of reconstruction used in the treatment of ACL injuries. ACL reconstruction uses athroscopically aided technique by making small cuts, have become a great choice $[6,7]$.

The goal of ACL reconstruction is to replace damaged ACL ligaments using graft materials that can restore the function of normal ligaments. Various studies on ligament reconstruction methods using various grafted material starting from synthetic materials, as well as derived from autograft tissue and even allografts have been carried out [7]. Arthroscopic reconstruction using an autograft or allograft tendon is currently become a gold standard treatment in ACL tears. The use of bone-patellar tendon-bone (BPTB), semitendinosis/gracilis hamstring autograft materials and allograft BPTB in reconstruction have become the main choices for ACL injury patients.

\section{Therapeutic Problems In ACl Management}

The development of arthroscopic techniques using both autograft and allograft still showed unsatisfactory results such as a ligament weakness, morbidity in the donor area and a long recovery period. The successful use of the graft is largely determined by the precise level of tension and fixation of the graft. ACL grafts that are too weak will not be able to restore knee stability. While ACL grafts that are too tight can cause failure of the transplant and limit the range of motion of the knee $[6,8]$. The condition of arthrofibrosis or soft tissue laxity is greatly influenced by the intrinsic condition of soft tissues. Patients with soft tissus that is too stiff may arise an extensive scar tissue accompanied with adhesions after the reconstruction. And conversely, patients with soft tissue that tend to loose have the potential to cause post-reconstruction weakness because scar tissue is formed slightly [7].

The incidence of osteoarthritis after ACL injury is also one of the other problems. The frequency of the early knee osteoarthritis and chronic pain after ACL injury are known to reach up to $20-50 \%$ of patients within $10-20$ years after injury [2,7]. Studies showed that this incident occurred in many young athletes who undergo reconstruction surgery.
The reconstruction did not prevent the development of osteoarthritis in the future [7].

The other thing that became a problem in ACL injury management is when the patient has a partial injury, where the reconstruction is not a treatment option. Wether intervention actions can be taken to optimize the healing process or the right choice is only the conservative management. Several studies have been developed to understand the biomolecular process and the development of various interventions, therapeutic modalities including the use of stem cells with various other biological materials that are known to have the ability to regenerate.

\section{TYPES OF STEM CELLS AND ITS APPLICATIONS}

Stem cells are undifferentiated types of cells that have the ability to differentiate into different cell types. These stem cells can be found int all stages of the development of organism. There are 2 types of stem cells, namely embryonic (pluripotent) and non-embryonic or adult (multipotent) stem cells. Embrionic stem cells (ESCs) is the stem cells that only found in the early stage of the organism development. This type is the only stem cell that has pluripotent properties, which has the ability to self-renew indefinitely. These stem cells can differentiate into cells of all germinal layers. ESC has shown promising potential in promoting regeneration in preclinical studies but their use in the clinic cannot be done because of the potential to become neoplasms and ethical problems while using ESCs. The concept of stem cell therapy currently focuses on the application of adult stem cells [9].

In process of therapy, adult stem cells has been widely used due to its ease of obtaining. Mesenchymal stem cells (MSC) is a non-hematopoietic adult stem cells that found in most connective tissues. Although included in adult stem cells, MSC has limited cellular differentiation capabilities. MSC has capabilities which can differentiate into various mesenchymal tissues such as tenocytes, osteoblasts, chondocytes and fat cells. Some of the advantages of MSC is easy to isolate. MSC can be isolated from various sources such as fat tissue (adipocyte MSC), synovial tissue or isolated from bone marrow (BM-MSC). Pre-clinical evidence shows that MSC has a beneficial effect through immunomodulatory and paracrine mechanisms. MSC triggers an inflammatory response and secretes bioactive molecules that helps in the healing process. MSC also has low immunogenicity, expresses very low MHC classes I and II, and does not activate allogenic lymphocytes. MSC applications are also simple, which can be given via injection of cell suspensions, or used in conjunction with other biomaterials. This makes the MSC as a preferred cell-based therapies [9-12]

\section{EFFECTIVENESS OF MESENCHYMAL STEM CELLS THERAPY IN ACL INJURY}

Evidence has shown that the use of MSC in ACL therapy in animals showed a significant tissue improvement. The presence of this reparative effect was demonstrated in a study by Kanaya who conducted direct BM-MSC intraarticular injection (exogenous administration) in mouse models with 
partial ACL rupture. This study has shown that BM-MSC could grow in intra-articular environments and improved the healing of partial ACL both bimechanically and histologically compared to groups without MSC. The ACL area in the BM-MSC group shows a better degree of histological feature and no retraction [13]. In line with Kanaya, the use of MSC culture and uncultured MSC in partial ACL therapy on Fischer 344 / Nslc mice also showed good effectiveness. MSC are able to differentiate into fibroblasts in the wound site area because the nature of tropism which mediated by high secretion of transforming growth factor- $\beta 1$ (TGF- $\beta 1)$ [14].

Although MSC have been known to play an important role in ligamentous regeneration due to their multipotential and trophic properties, because ligament healing is a complex physiological process repair of injured ACL with MSC therapy alone give less than optimal results. Damage to the ligament tissue after ACL rupture due to high strain results in reducing the scaffold required. the absence of this scaffold is an important cause of inhibition of tissue regeneration in joints, such as ACL or meniscus. Scaffold can provide homing signals to MSC and help the attachment, proliferation process and cell differentiation. This reduced scaffold is associated with decreased levels of inflammatory cytokines needed for healing responses such as fibrinogen, PDGF, TGF- $\beta$ and FGF $[4,8,15]$.

Studied using MSC and silk scaffold to assess ACL regeneration were carried out in 2008 by creating the scaffold resemble the ECM ligamentous structure. In vitro results showed that MSC with scaffold was able to proliferate rapidly and produced a lot of collagen. Then MSC/scaffold is implemented in vivo. After 24 weeks, MSC was distributed in the area of the regenerated ligament and differentiated into fibroblast morphology. ECM components that are widely produced were collagen type I, III and tenascin-C. Bone ligament insertion which underwent reconstruction resembling the original structure of the ACLbone insertion. Tensile strength were also significantly higher than controls [16].

Another study compared the regeneration process in complete rupture of ACL between MCS with type I collagen scaffold group, type I collagen scaffold alone without MSC and suture procedure group. The result showed that MCS with type I collagen scaffold group could regenerate into normal ACL tissues in $33 \%$ of cases as indicated by histological features of organized collagen tissue with peripheral blood vessel growth. However, this was not found in the group that only received therapy with sutures alone, or with type I collagen scaffold alone without MSC [17].

The addition of various growth factors have also been applied to increase the proliferation and the differentiation of MSC and also extracellular matrix deposition on the scaffold. One of the study of TGF $\beta 1 /$ VEGF joint expression in the remodeling process of ACL graft showed that the expression of TGF $\beta 1$, in TGF $\beta 1 / V E G F-t r a n s f e c t e d ~ B M S C$, could accelerate the remodelling of reconstructed ligaments. Remodelling of the graft after ACL reconstruction requires a long recovery period before being able to withstand physiological loads. Graft revascularization is very important in the remodeling process. TGF $\beta 1 /$ VEGF-transfected BMSC were significantly able to trigger the occurrence of angiogenesis in reconstructed ligament at 3, 6, 12 weeks and optimum mechanical ability was achieved at 24 weeks [18].

The application of PRP, which contains of various growth factors, was also reported. In vitro studies of the application of PRP in anterior cruciate ligament reconstruction in a rabbit model revealed that collagen I, osteocalcin, and osteopontin expression were found to be higher in the BMSC group cultured with PRP than in the PRP group or control group only. More mature and newly formed bone tendon formation is obtained at the bone tunnel walls [19].

Several studies have also demonstrated that mechanical stimulation of MSC cultured on special biomaterials could induce their differentiation into ligamentous fibroblasts. One study that assessed the continuous passive motion (CPM) effect on co-culture of bone marrow mesenchymal stem cells (BMSC) and tendon cells (TCs) therapy in animal models with ACL showed that CPM significantly improved the healing of proliferation of tendons, differentiation of more fibrocartilage, and up-regulated gene expressions of Collagen I, alkaline phosphatase, osteopontin, Tenascin $\mathrm{C}$ and tenulinulin at the tendon-bone junction [20].

\section{SAFETY ISSUES IN MSC THERAPY}

Problems related to immunogenicity of MSC are of concern in the application of MSC as a therapeutic agent. A study by Schu stated that MSC is not fully immunoprivileged in allogenic immunocompetent hosts. Although the number of CD4+ $\mathrm{T}$ cell activation and humoral immune response is substantially reduced, a single injection of allo-MSC is sufficient to induce the production of complement-fixing alloantibodies and facilitate rapid rejection of subsequent injections [10].

In contrast to Schu, non-autologous studies of human mesenchymal cord stem cells (hUCB-MSC) in blood transplants in ACL reconstruction in animal models have proven that they could be applied without causing any initial immune rejection. There was also an increase in tendon bone healing through extensive fibrocartilage formation with a higher histological score compared to the control group [21]. Systematic review study that assessed the side effects of allogenic MSC therapy also showed that there were no acute toxicity events other than transient fever. This supports the idea that MSC is immunoprivileged, which can be explained by the low expression of the MHC protein and the molecule of T-cell modulators. These results provide that this innovative therapy is safe [12].

Although MSC has been known to have potential therapeutic value, the exact mechanism of MSC in improving ligament healing remains unclear. Study of allogenic MSC in ligament injuries indicated that dose could affect cellular response and cytokine expression during healing. High doses of MSC had the effect of early healing (day 5) both in cell and cytokine changes, but cause poor results. Whereas low doses of MSC showed slight changes during initial healing, but gave an increase in mechanical function (day 14). This 
study showed that a large number of MSC are not always better when using allogenic MSC and therefore the dosage must be strictly checked for each application. These possibilities can occur due to the influence of MSC sources (including auto-versus allo-MSC), the number of MSC, animal models and type of tissues. In short, dosage needs to be considered in each injury model because MSC has the ability to change processes and results both positively and negatively during healing [11].

\section{VIII.CONCLUSION}

The anterior cruciate ligament has limited ability to recover when an injury occurs. Current non-surgical treatment procedures often provide unfavorable results when applied in cases of ACL rupture. Therefore, surgical reconstruction of ACL is the main choice therapy to obtain knee stability. But in reality, ACL reconstruction often provides unsatisfactory long-term results in addition to the many complications that occur due to surgery. Efforts to increase the degree of healing in ACL reconstruction are very important to facilitate an early and aggressive rehabilitation and regain capacity as before.

In the latest ACL reconstruction techniques, many preclinical experimental evidence suggests that healing results can be further enhanced by modulation of biological agents to improve intraarticular and intraosseous healing. Current evidence suggests that the addition of MSCs can be an effective tool for achieving regenerative ligaments. This is most likely because stem cells have regeneration potential. But apparently the results can vary because ligament healing is a complex physiological process involving four different factors for optimal tissue restoration, namely the presence of MSC, scaffold, growth factors and mechanical stimulus.

The approach of using growth factors, stem cells, scaffold and mechanical stimulus effects has also been widely investigated in healing ACL injuries and providing a significance results in tissue repair and demonstrating safety in their applications. But because most of this research is still in preclinical animal testing, further research is needed to see whether clinical trials are useful. Therefore, translational research to bring preclinical findings into good quality clinical trials in the future becomes very important.

\section{REFERENCES}

[1] S. Neeraj, "International epidemiology of anterior cruciate ligament injuries," Ortho Res Online J., 2018, 1(5).

[2] A. M. Joseph, C. L. Collins, N. M. Henke, E. E. Yard, S. K. Fields, R. D. Comstock, "A Multisport epidemiologic comparison of anterior cruciate ligament injuries in high school athletics," Journal of Athletic Training, 2013, 48(6), pp.810-7.

[3] B. Moses, J. Orchard, J.Orchard, "Systematic review: annual incidence of ACL injury and surgery in various populations," Research in Sports Medicine, 2012, 20 (3-4).

[4] P. S. Pastides, W. S. Khan, "Cell-based therapies in musculoskeletal injuries: the evolving role of bone marrow-derived mesenchymal stem cells," British Journal of Medicine \& Medical Research, 2011, 1(4), pp. $486-500$

[5] J. Xie, C. Wang, L. Yin, C. M. Xu, Y. Zhang, K. L. P. Sung, "Interleukin-1 beta influences on lysyl oxidases and matrix metalloproteinases profile of injured anterior cruciate ligament and medial collateral ligament fibroblasts,“ Int Orthop, 2013, 37(3), pp. 495-505.

[6] P. J. McMahon (Ed), Current diagnosis \& treatment in sports medicine, Unites States, US: McGraw Hill, 2007.

[7] P. Brukner, K. Khan, Brukner \& Khan's clinical sports medicine, 4th ed, Australia, North Ryde: McGraw Hill, 2007.

[8] D. Kouroupis, S. M. Churchman, P. V. Giannoudis, E. Jones, "Mesenchymal stem cell applications for ligament repair after joint trauma," J Clin Exp Pathol, 2014, 4 (186).

[9] A. G.-Alvarez, A. Schmitt, R. P. Russell, A. B. Imhoff,, S. Buchmann, "Growth factor delivery vehicles for tendon injuries," Muscles, Ligaments and Tendons Journal, 2014, 4 (3), pp.378-85.

[10] S. Schu, M. Nosov, L. O’Flynn, G. Shaw, O. Treacy, F. Barry, M. Murphy, T. O'Brien, T. Ritter, "Immunogenicity of allogeneic mesenchymal stem cells," J. Cell. Mol. Med., 2012, 16(9), pp. 2094103.

[11] E. E. Saether, C. S. Chamberlain, E. M. Leiferman, J. R. KondratkoMittnacht, W. J. Li, S. L. Brickson, R. Vanderby, "Enhanced medial collateral ligament healing using mesenchymal stem cells: dosage effects on cellular response and cytokine profile," Stem Cell Rev., 2014, 10(1), pp.86-96.

[12] M. M. Lalu, L. McIntyre, C. Pugliese, D. Fergusson, B. W. Winston, J. C. Marshall, J. Granton, D. J. Stewart, "Safety of cell therapy with mesenchymal stromal cells (SafeCell): a systematic review and metaanalysis of clinical trials," PLoS One, 2012, 7(10), pp. e47559.

[13] A. Kanaya, M. Deie, N. Adachi, M. Nishimori, S. Yanada, M. Ochi, "Intra-articular injection of mesenchymal stromal cells in partially torn anterior cruciate ligaments in a rat model," Arthroscopy, 2007, 23, pp. 610-7.

[14] K. Oe, T. Kushida, N. Okamoto, M. Umeda, T. Nakamura, S. Ikehara, et al, "New strategies for anterior cruciate ligament partial rupture using bone marrow transplantation in rats," Stem Cells Dev, 2011, 20, pp. 671-9.

[15] P. Y Mengsteab, L. S Nair, C. T Laurencin, "The past, present and future of ligament regenerative engineering," Regen. Med., 2016, $11(8)$, pp. 871-81.

[16] H. Fan, H. Liu, E. J. Wong, S. L. Toh, J. C. Goh, "In vivo study of anterior cruciate ligament regeneration using mesenchymal stem cells and silk scaffold," Biomaterials, 2008, 29(23), pp. 3324-37.

[17] D. Figueroa, M. Espinosa, R. Calvo, M. Scheu, A. Vaisman, M. Gallegos, P. Conget, "Anterior cruciate ligament regeneration using mesenchymal stem cellsand collagen type I scaffold in a rabbit model," Knee Surg Sports Traumatol Arthrosc, 2014, 22(5). pp. 1196-202

[18] X. Wei, Z. Mao, Y. Hou, L. Lin, T. Xue, L. Chen, H. Wang, C. Yu, "Local administration of TGFß-1/VEGF165 gene-transduced bone mesenchymal stem cells for Achilles allograft replacement of the anterior cruciate ligament in rabbits," Biochem Biophys Res Commun, 2011, 406(2), pp. 204-10.

[19] C. Teng, C. Zhou, D. Xu, F. Bi, "Combination of platelet-rich plasma and bone marrow mesenchymal stem cells enhances tendon-bone healing in a rabbit model of anterior cruciate ligament reconstruction," J Orthop Surg Res, 2016, 11(1), pp. 96.

[20] F. Song, D. Jiang, T. Wang, Y. Wang, F. Chen, G. Xu, Y. Kang, Y. Zhang, "Mechanical loading improves tendon-bone healing in a rabbit anterior cruciate ligament reconstruction model by promoting proliferation and matrix formation of mesenchymal stem cells and tendon cells," Cell Physiol Biochem, 2017, 41(3)8 pp. 875-89.

[21] K. M. Jang, H. C. Lim, W. Y. Jung, S. W. Moon, J. H. Wang, "Efficacy and safety of human umbilical cord blood-derived mesenchymal stem cells in anterior cruciate ligament reconstruction of a rabbit model: new strategy to enhance tendon graft healing," Arthroscopy, 2015, 31(8), pp. 1530-9. 